\begin{tabular}{|c|c|}
\hline \multirow{3}{*}{ 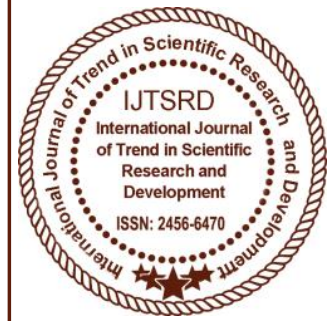 } & $\begin{array}{l}\text { International Journal of Trend in Scientific } \\
\text { Research and Development (IJTSRD) }\end{array}$ \\
\hline & UGC Approved International Open Access Journal \\
\hline & ISSN No: 2456 - 6470 | www.ijtsrd.com | Volume - 1 | Issue - 5 \\
\hline
\end{tabular}

\title{
Smart Attendance System using Raspberry Pi
}

\author{
Pradeep Kumar M S \\ Asst. Professor, Dept. of TCE, DBIT College, \\ Bangalore, Karnataka, India \\ Indumati $T$ \\ Asst. Professor, Dept. of CSE, EWIT College, \\ Bangalore, India
}

\author{
Dr. K. Suresh \\ Principal, SDMIT college, Ujire, Karnataka, India \\ Kishor kumar \\ Asst. Professor, Dept. of TCE, DBIT College, \\ Bangalore, Karnataka, India
}

\begin{abstract}
Biometric authentication is one of the most popular and accurate technology. Now days, it is used in many real time applications. However, recognizing finger prints in Linux based embedded computers (raspberry pi) is still a very complex problem. This entire work is done on the Linux based embedded computer called raspberry pi, in which database creation, fingerprint reader access, authentication and recognition using python were entirely done on raspberry pi This paper discusses on the standardized authentication model which is capable of extracting the finger prints of individual and store that in database. Then the use of final finger print to match with others in finger prints present in the database to show the capability of this model and also updating the database obtained to the organisation by creating an application through cloud.
\end{abstract}

Keywords: finger print authentication, python, raspberry pi

\section{INTRODUCTION}

The human body has the privilege of having features that are unique and exclusive to each individual. This exclusivity and unique characteristic has led to the field of biometrics and its application in ensuring security in various fields with various embedded controllers and embedded computers. Biometrics has gained popularity and has proved itself to be a reliable mode of ensuring privacy, maintaining security and identifying individuals. It has wide acceptance throughout the globe and now is being used at places like airports, hospitals, schools, colleges, corporate offices etc.

Biometrics is the study of identifying a person by their physical traits that are inherent and unique to only the person concerned. Biometric identification include fingerprint verification, palm geometry, face recognition, iris recognition, etc. The above mentioned techniques work with different levels of functionality and accuracy.

Accuracy and reliability are the two most important parameters when it comes to biometric applications and that too with advanced embedded computers. Fingerprint verification is one of the oldest known biometric techniques known but still is the most widely used because of its simplicity and good levels of accuracy. It's a well known fact that every human being is born with a different pattern on the fingers and this feature is exploited to identify and differentiate between two different persons that is what the factor which helped to initiate the model.

\subsection{LITERATURE SURVEY}

Sonam Shukla, Pradeep Mishra suggested increasing the Accuracy of an Existing Fingerprint Recognition System Using Adaptive Technique, in this approach developer mainly focusing on Integrated Automated Fingerprint Identification Service (IAFIS) of the most famous police agencies. [1] They extracted fingerprint pattern is characterized by a set of ridgelines that 
often flow in parallel, but intersect and terminate at some points. The uniqueness of a fingerprint is determined by the local ridge characteristics and their relationships. Main drawback of this model is this approach is not so apt for real time applications but the accuracy of system is highly adaptable. Most automatic systems for fingerprint comparison are based on minutiae matching.

Le Hoang Thai and Ha Nhat Tam in 2010 suggested Fingerprint recognition using standardized fingerprint model, now a days, fingerprint recognition is one of the most Important biometric technologies based on fingerprint distinctiveness, [2] in this approach they focused on improving the quality of fingerprint images. In fingerprint recognition process, the important step which affects on system accuracy is matching between template and query fingerprint

This approach functions based on the uniqueness of each person and it integrating the biometric device to transmit the information obtained in this approach they are using fingerprint extraction and matching algorithm and they maintaining the database to authenticate the person who approaching for the access through the

On line web page created in the local server.

\section{SYSTEM OUTLINE:}

\subsection{HARDWARE ARCHITECTURE:}

This proposed system consists of raspberry pi and fingerprint module. This system provides the features of real time authentication facilities

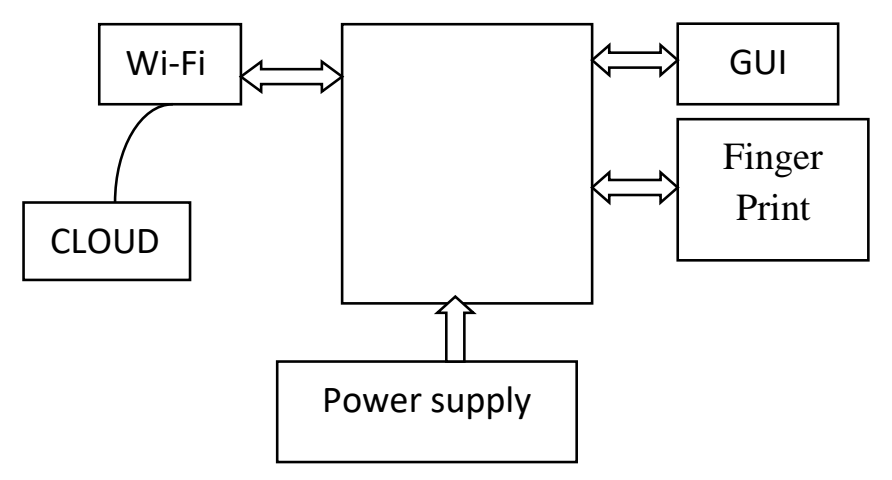

Fig 1: Proposed System Block Diagram

\subsubsection{RASPBERRY PI:}

Hardware is a physical device that can be touched or held, like a hard drive or a mobile phone. Software can be thought of as a program or a collection of programs that instruct a computer on what to do and how to do it. Below is an image of the Raspberry Pi which describes some of the components that make up the hardware.

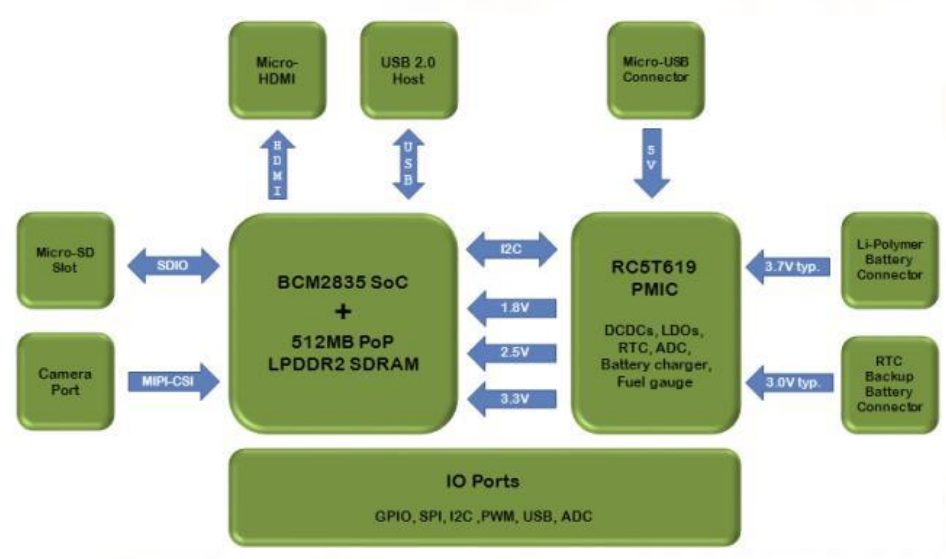

Fig.2. Hardware Components of Raspberry Pi

Fig. 2 shows the hardware description of raspberry pi. The real time vehicle tracking system uses the GPIO pin, Micro SD slot, USB port and Micro USB connector. The GPIO pins are used for serial communication for interfacing GSM and GPS. It uses 8GB SD for installing the Raspbian OS and for storage. The USB port is used for connecting keyboard, mouse, dongle and pen drive. The power supply is given through USB connector.

\subsubsection{FINGERPRINT READER:}

The R305 is the module which is used in the project. This module supports both windows and Linux based operating system. This self-contained module optically scans the fingerprint when the user touches the glowing window.

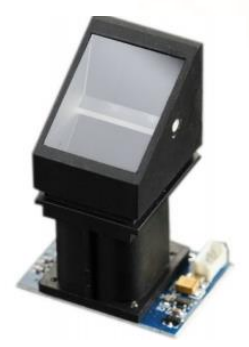

Fig 3: Finger print module 
Optical technology gives the highest quality fingerprint templates and reliability along with the raspberry pi embedded computer.

The device can automatically control calibration, encryption, and data transfer through the USB interface.

This module is found to be more reliable for all kind of OS, even its easy to interface with raspberry pi.The R305 Module and digital Persona Fingerprint Recognition Engine have an unmatched ability to authenticate even the most difficult fingerprints accurately and rapidly.

\subsubsection{GUI (Graphical User Interface):}

The graphical user interface (GUI) is a type of user interface that allows users to interact with electronic devices through graphical icons. Visual indicators such as secondary notation, instead of text-based user interfaces, typed command labels or text navigation.

\subsubsection{POWER SUPPLY:}

Model uses 5 volt power supply and has a ability to drive 2.5 amperes of current.

\subsection{SOFTWARE ARCHITECTURE:}

For this project the used softwares are like PHP, postgresql, opencv (python script). The configuring procedures, saving and retrieval of fingerprint templates procedures are mentioned in the upcoming chapters.

\subsubsection{SOFTWARE- ALGORITHM:}

The software algorithm of authentication system is described here,

Step 1: Initialize the device by switching on the power supply.

Step 2: Run the processor.

Step 3: Connect the fingerprint module.

Step 4: Open the fingerprint_demo gui.

Step 5 : Read and save the image template.

Step 6: Covert the image template in to binary template

Step 7: Save the obtained template in the MSQL database

Step 8: Now comparison between various templates can be carried out using the python script.

Step 9: The accurate matching points will obtain between the images.
Step 10: After matching and comparison, the overall database is stored in excel file to upload it to the cloud.

\subsection{IMPLEMENTATION PROCEDURE:}

\subsubsection{FPRINT DEMO: GRAPHICAL:}

\section{DEMONSTRATION APPLICATION:}

This proposed system is implemented in a Linux based GUI application, fprint demo and fingerprint model, with the intention of using this to demonstrate the functionality of the finger print device along with raspberry pi embedded computer. fprint demo offers an interface for enrolling fingers and deleting enrollment data for previously enrolled fingers with the help of fprint GUI, an interface for verifying a specific finger and an Interface for identifying a finger from a selection of previously enrolled fingerprint data.

\subsubsection{CONVERTING THE TEMPLATE IN TO BINARAIZED TEMPLATE}

This GUI is capable of converting the fingerprint template in to binary template. The imaging performance of this device is excellent. The returned images are large and find a large number of minutiae, leading to suitably distinct scores for matching/non-matching fingers. Fig 4 shows an image returned by this driver and its binarized form with minutiae plotted with the help of raspberry pi embedded computer.

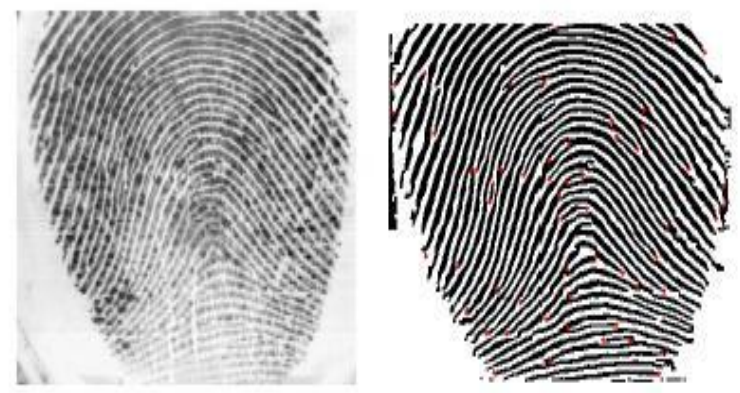

Fig 4: Normal and binarized template

Fig 5 given below shows mismatched fingerprints of different persons the python program is running as the background process. Thus the below figure shows the mismatched characteristics of two different fingers, while comparing the same fingers I got the matching points almost everywhere in finger template. 

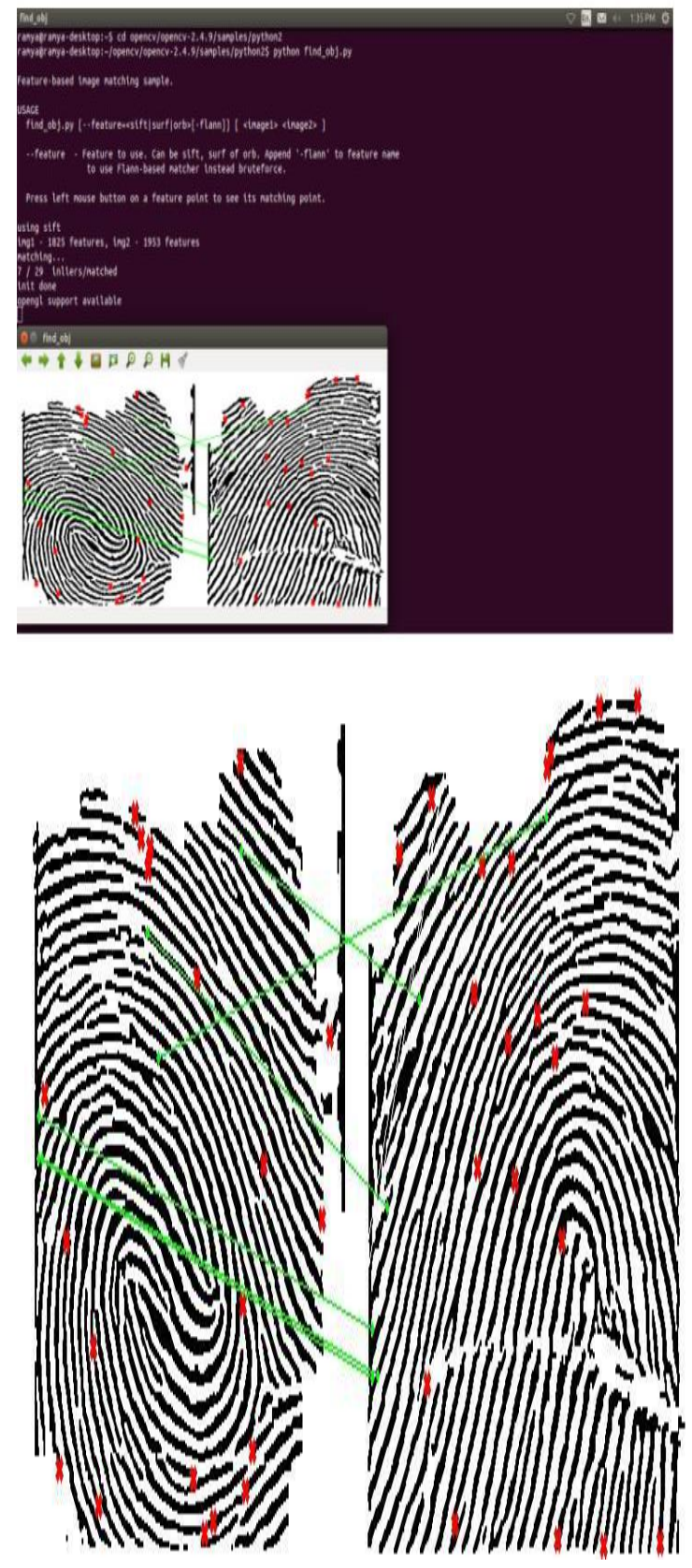

Fig 5: Mismatched finger print

Fig 6 given below shows exactly matched fingerprints of a person the python program is running as the background process The green coloured lines connecting two templates showing the number of matching points in the templates The below experimental results clearly states the ability of the proposed design to distinguish the matched and mismatched fingers.

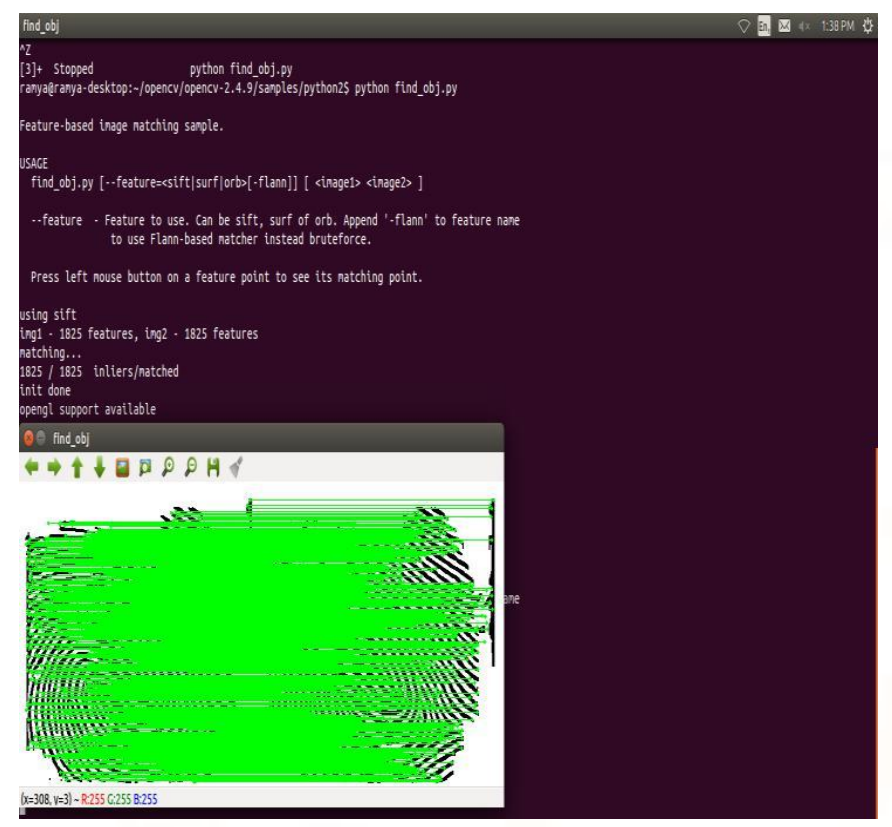

Fig 6 . Matched fingers print

\section{EXPREIMENTAL RESULTS:}

After extracting the fingerprint template using finger print demo GUI, Then comparison has been carried out with the help of open cv programming tool with python script and generate the excel file database to upload it to cloud.
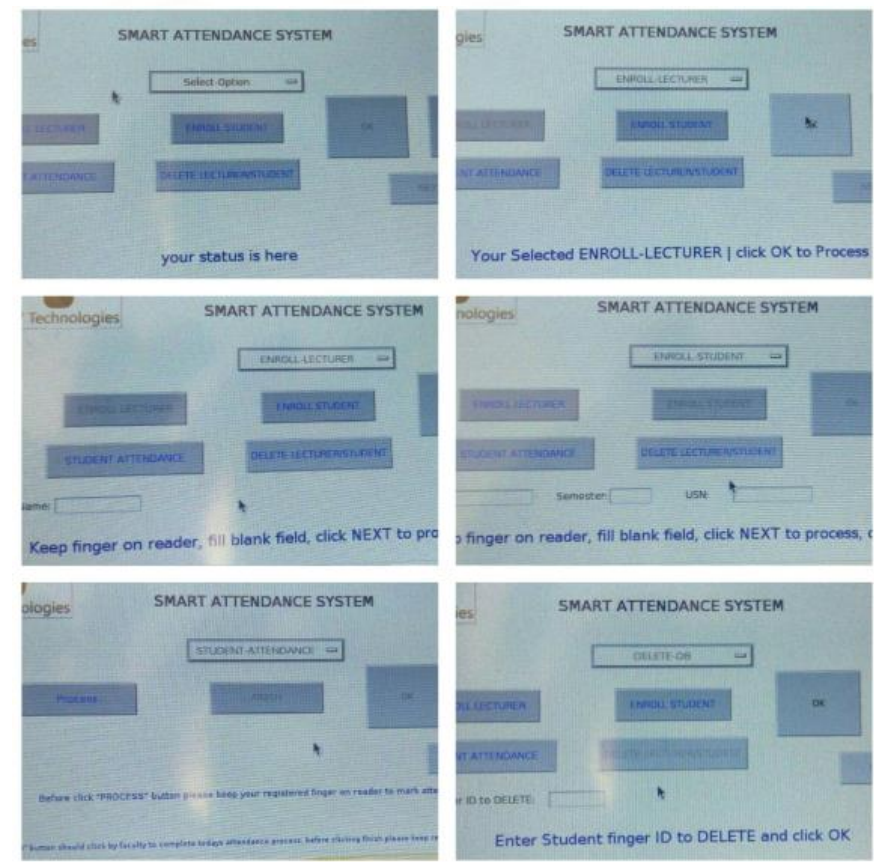


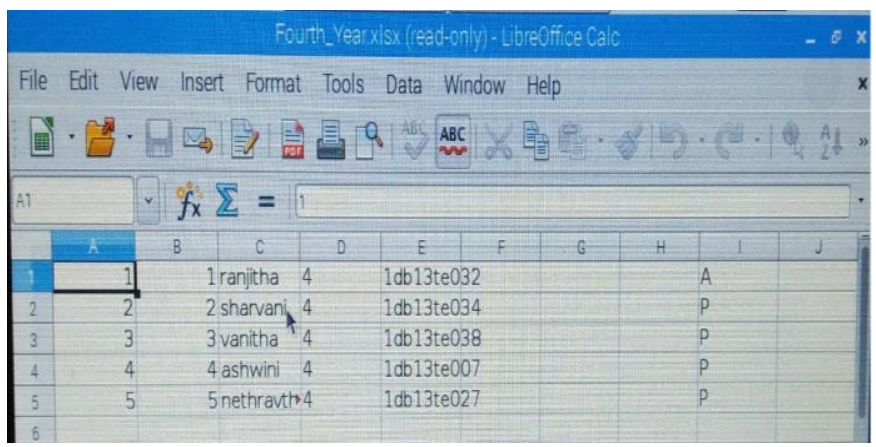

\section{CONCLUSIONS AND FUTURE WORK:}

Fingerprint recognition is one of the most popular and accurate Biometric technologies. Nowadays, it is used in many real time applications. However, recognizing fingerprints in Linux based recognition using python were entirely done on raspberry pi This paper discusses on the standardized fingerprint model which is used to synthesize the template of fingerprints. In this model, after pre- processing step, find the transformation between templates, adjust parameters and synthesize fingerprint. Then the use of final fingerprint to match with others in fingerprints present in the database to show the capability of this model. Embedded computers (raspberry pi) are still a very complex problem. This entire work is done on the Linux based embedded computer called raspberry pi , in which database creation and management, fingerprint reader access, authentication and fine tuning has to be done to improve the accuracy of the system and developing the system for real time higher end application development using cloud created are done. In future this single device can also be implemented, and used by many lecturers.

\section{ANDROID}

Android is a mobile operating system developed by Google, based on the Linux kernel and designed primarily for touch screen mobile devices such as smart phones and tablets. Application is created to see the student attendance report for organisation use.

\section{REFERENCES}

1) Sonam Shukla, Pradeep Mishra [2012],"Increasing The Accuracy Of An Existing Recognition System Using Adaptive Technique" International Journal of Advanced Research in Computer Science and Software Engineering, Volume 2, Issue 6, PP 52-57.
2) Le Hoang Thai and Ha Nhat Tam [2010]," Fingerprint recognition using Standardized M fingerprint model" IJCSI International Journal of Computer Science Issues, Vol. 7, Issue 3, PP 1116.

3) Mukesh Kumar Thakur, Ravi Shankar Kumar, Mohit Kumar, Raju Kumar [2013],"Wireless Fingerprint Based Security System Using Zigbee Technology" International Journal of Inventive Engineering and Sciences (IJIES), ISSN:23199598, Volume-1, Issue-5, PP 14-17

4) Arun, Emmanuel., Diwakar\& Rajeswari Automated attendance system using biometrics with Embedded web serverll Graduate Research in Engineering and Technology (GRET): An International Journal, page No-(54to57).

5) Karthik Vignesh E, Shanmuganathan S, A.Sumithra S.Kishore and P. Karthikeyan -A Foolproof Biometric Attendance Management System\|I International Journal of Information and ComputationTechnology.ISSN:0974-2239. 